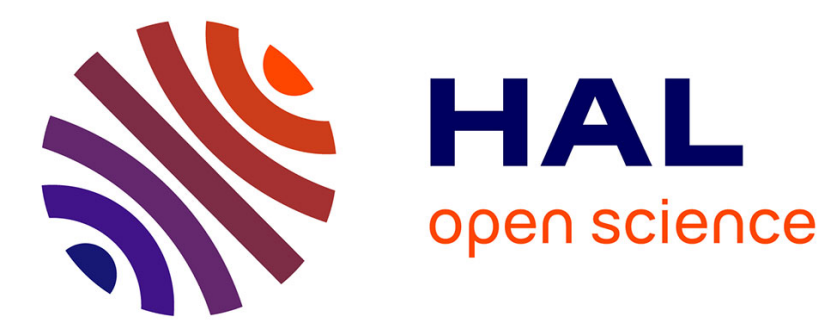

\title{
Différenciation et caractérisation de deux unités d'un système plage: cordon de galets et bas de plage sableux. Le cas des Bas-Champs de Cayeux (Somme) (Note)
} Franck Dolique

\section{- To cite this version:}

Franck Dolique. Différenciation et caractérisation de deux unités d'un système plage: cordon de galets et bas de plage sableux. Le cas des Bas-Champs de Cayeux (Somme) (Note). Méditerranée: revue géographique des pays méditerranéens, 1999, 93 (4), pp.69-72. 10.3406/medit.1999.3135 . hal02542772

\section{HAL Id: hal-02542772 \\ https://hal.science/hal-02542772}

Submitted on 8 Feb 2021

HAL is a multi-disciplinary open access archive for the deposit and dissemination of scientific research documents, whether they are published or not. The documents may come from teaching and research institutions in France or abroad, or from public or private research centers.
L'archive ouverte pluridisciplinaire HAL, est destinée au dépôt et à la diffusion de documents scientifiques de niveau recherche, publiés ou non, émanant des établissements d'enseignement et de recherche français ou étrangers, des laboratoires publics ou privés. 


\section{Différenciation et caractérisation de deux unités d'un système plage}

: cordon de galets et bas de plage sableux. Le cas des Bas-

Champs de Cayeux (Somme) (Note)

\section{Franck Dolique}

\section{Citer ce document / Cite this document :}

Dolique Franck. Différenciation et caractérisation de deux unités d'un système plage : cordon de galets et bas de plage sableux. Le cas des Bas-Champs de Cayeux (Somme) (Note). In: Méditerranée, tome 93, 4-1999. Dynamiques naturelles et gestion des espaces littoraux. pp. 69-72;

doi : https://doi.org/10.3406/medit.1999.3135

https://www.persee.fr/doc/medit_0025-8296_1999_num_93_4_3135

Fichier pdf généré le 25/04/2018 


\section{Différenciation et caractérisation de deux unités d'un}

\section{système plage : cordon de galets et bas de plage sableux.}

\section{Le cas des Bas-Champs de Cayeux (Somme)}

\section{Franck DOLIQUE*}

Depuis les vingt dernières années, la description des états de plages est passée d'une caractérisation qualitative à une approche plus quantitative, absolument nécessaire pour appréhender les évolutions morphosédimentaires à court terme et sur une échelle spatiale réduite. Cette approche a généré un nombre toujours croissant d'indices et de paramètres semi-quantitatifs ayant un intérêt essentiellement pédagogique et démonstratif. Dans ce cadre, il apparaissait intéressant de tester et calibrer les paramètres morphodynamiques les plus usités dans la littérature, notamment les paramètres de déferlement, sur un site dont le profil de plage présente une forte dichotomie topographique entre le haut de plage et le bas de plage, et ainsi comparer facilement l'effet de la topographie de la plage sur le déferlement.

C'est pour cette raison que le site des BasChamps de Cayeux, sur le littoral picard (fig. 1), a été choisi car cette côte présente une morphologie idéale pour suivre l'évolution du type de déferlement par rapport à la topographie de la plage et en fonction de la marée, surtout sur ce secteur macrotidal. Nous trouvons en effet sur ce site un massif cordon de galets au profil raide $\left(16^{\circ}\right)$ en haut de plage, qui repose sur une surface sableuse à pente très faible $\left(0,9^{\circ}\right)$ qui constitue le bas de plage (fig. $2 a$ et b).

\section{Caractéristiques des domaines dissipants et réfléchissants}

L'approche morphodynamique reconnaît une diversité au sein des plages essentiellement en fonction de la manière dont la houle interagit avec le substrat. Il ressort de cette approche que le comportement physique d'une plage peut être caractérisé en fonction du degré de réflexion ou de dissipation de l'énergie de la houle. Cette classification

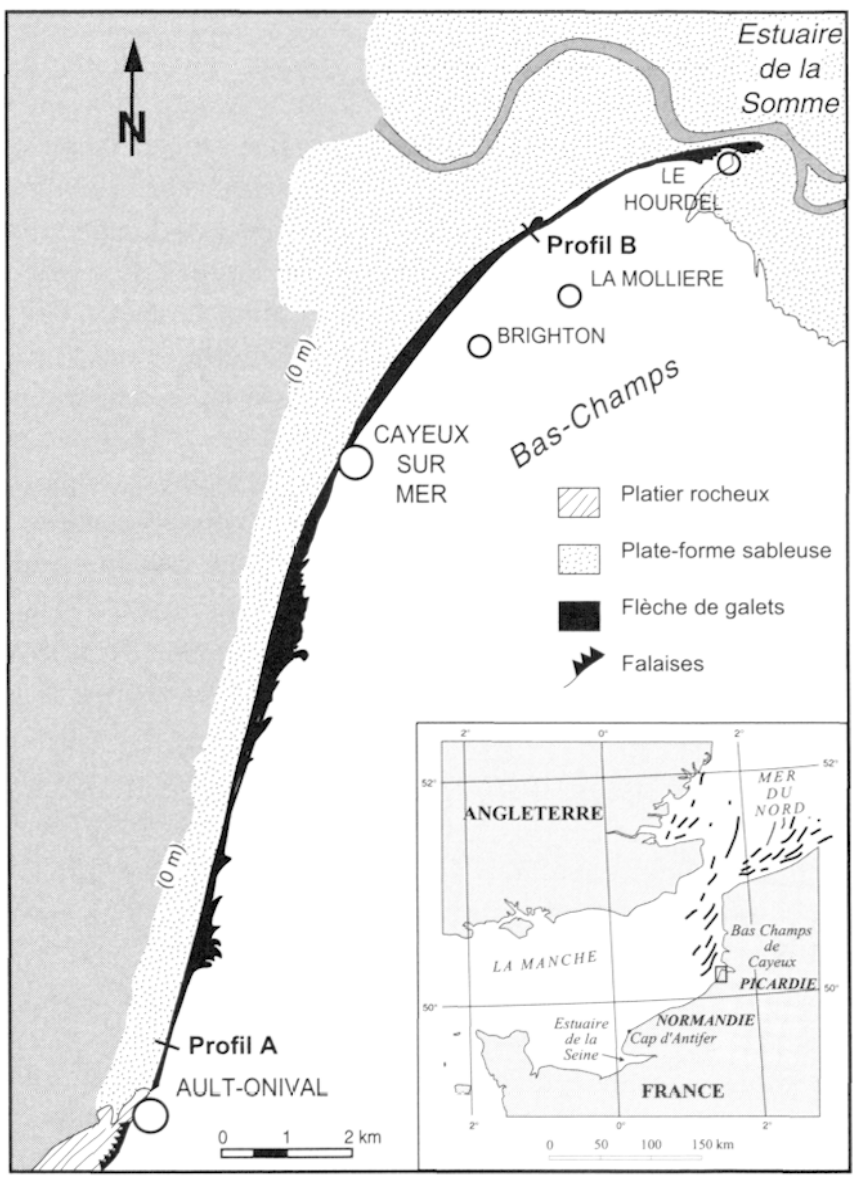

FIG. 1 - LOCALISATION

s'inspire en fait de la notion de «différence de phase» employée pour la première fois par P.H. KEMP (1961) et P.H. Kemp et D.T. Plinston (1968). Cet indice permet de différencier une plage en prenant en compte la durée du jet de rive (Ts) par rapport à la période de la houle $(\mathrm{T})$. Les critères de classification se sont par la suite affinés grâce à un continuum de publications (ShORT et Hesp, 1982 ; Wright et ShORT, 1983, 1984 ; CARTER, 1988).

* Université du littoral, Laboratoire de géomorphologie dynamique et aménagement des littoraux JE 2208 MENESR, Dunkerque. 


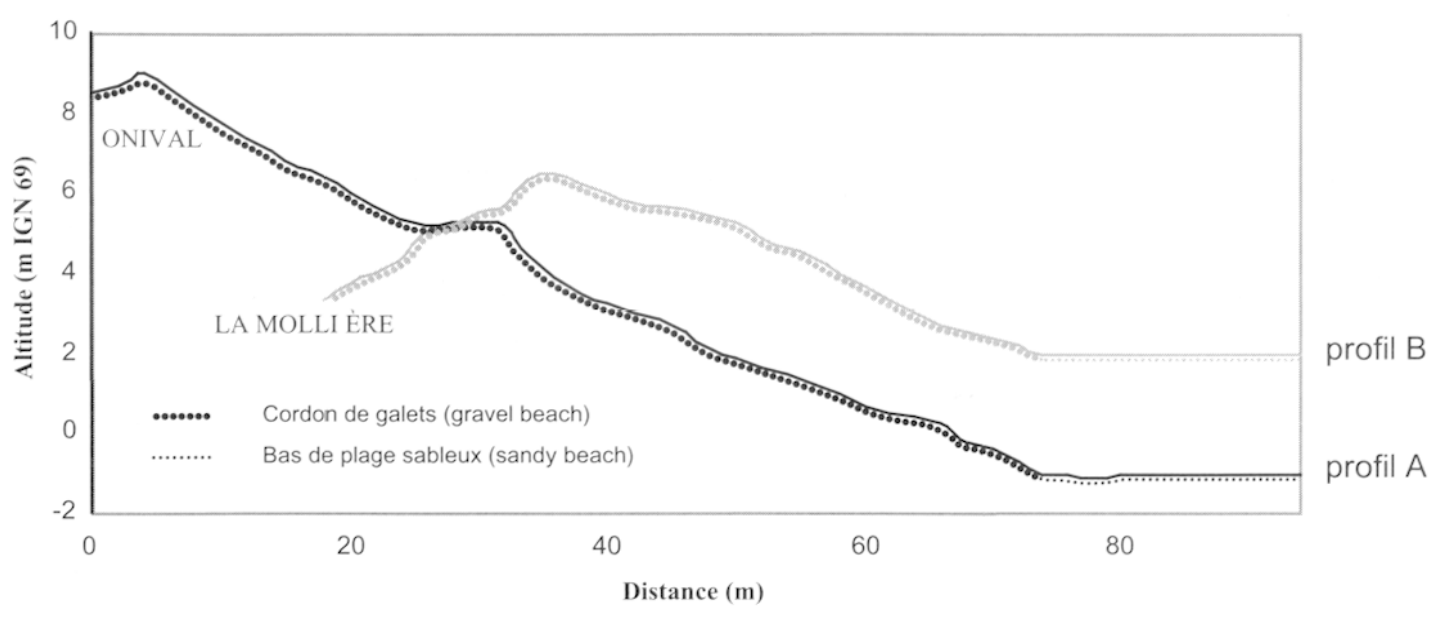

FIG. 2 - PROFILS MONTRANT L'OPPOSITION MORPHOLOGIQUE DE LA PLAGE

Les plages dissipantes sont caractérisées par un régime énergétique où les vagues, généralement de type déversant (spilling breaker), déferlent selon des périodes de jet de rive supérieures à 60 secondes et selon un angle d'attaque «normal» (proche de $0^{\circ}$ ) sur un estran large et peu pentu propice à la réfraction, d'où cet angle d'approche. Le régime de houle est caractérisé par des cascades d'énergie vers des ondes d'infragravité, à fréquence nettement plus basse (généralement supérieure à 15 secondes) que celle des vagues incidentes de gravité (fréquence observée). La pente de la plage est généralement inférieure à $1^{\circ}$ avec un profil plat ou très faiblement pentu. Les morphologies de barres sont dominantes avec des recyclages sédimentaires transversaux. On peut également noter la présence de «mégacroissants», qui sont des croissants de plage de grande longueur d'onde (>75 m).

Les plages réfléchissantes sont caractérisées par des déferlements en volute (plunging breaker) ou à effondrement (collapsing breaker). Leur angle d'approche est généralement oblique du fait d'une faible réfraction de la houle par un estran étroit et pentu. La pente de la plage est généralement supérieure à $3^{\circ}$ et le profil est raide ou concave. Le régime énergétique est dominé par des fréquences incidentes avec excitation de fréquences harmoniques (correspondant à une demi-période : $\mathrm{T} / 2$ ) et subharmoniques (deux à quatre fois la période : 2 à 4T). Cela se traduit concrètement par l'observation d'ondes de réflexion en interférence avec la houle incidente. La période du jet de rive est comprise entre 5 et 10 secondes. La morphologie est dominée par une absence de barres parallèles à la côte et par la présence de bermes et de croissants de faible amplitude.

Ce type de modèle, réalisé à l'origine à partir de milieux microtidaux, ne couvre pas toute la gamme de morphologies de plages. Il s'agit de ne pas généraliser car des exceptions peuvent toujours compliquer le schéma établi. Ces modèles constituent néanmoins des outils très intéressants permettant de visualiser des plages dans un continuum spatiotemporel morphodynamique.

\section{L'approche morphodynamique semi- quantitative}

Dans un souci démonstratif et pédagogique, l'approche morphodynamique a généré un faisceau de paramètres et d'indices semi-quantitatifs, élaborés de façon plus ou moins empirique et qui sont très utilisés et développés par les géomorphologues, géologues, ingénieurs et hydrodynamiciens. Détaillons ici les formulations utilisées :

Surf scaling parameter (paramètre d'échelonnement de barre) :

$\varepsilon=(\mathrm{ab}) \omega^{2} /\left(\mathrm{g} \tan ^{2} \beta\right)$

si $\varepsilon$ est $<2,5$ : la plage est réfléchissante

si $\varepsilon$ est $>30$ : la plage est dissipante.

Surf similarity (paramètre de réplication de barre) $\xi=\tan \beta /(\mathrm{Hb} / \mathrm{Lo})^{0.5}$

si $\xi$ est $>1$ : la plage est réfléchissante

si $\xi$ est $<0,23$ : la plage est dissipante phase)

Phase difference (paramètre de différence de

Ts/T

si $\mathrm{Ts} / \mathrm{T}<0,5$ : la plage est réfléchissante

si $\mathrm{T} s / \mathrm{T}>1$ : la plage est dissipante

Breaker coefficient (coefficient de seuil de déferlement)

$\mathrm{Bo}=\mathrm{Hb} / \mathrm{gT}^{2} \tan \beta$

si Bo<0,1 : le déferlement est de type plongeant, caractéristique des plages réfléchissantes

si Bo $>0,1$ : le déferlement est de type déversant. caractéristique des plages dissipantes.

Gamma parameter (paramètre gamma) $\gamma=\mathrm{Hb} / \mathrm{db}$

si $\gamma<0,65$ : le déferlement est déversant

si $\gg 0,65$ : le déferlement est plongeant 
Avec les variables suivantes qui composent les formulations :

Hb : Hauteur de la houle au déferlement (en m)

ab : amplitude du jet de rive (en $\mathrm{m}$ )

db : hauteur d'eau au point de déferlement (en $\mathrm{m}$ )

$T$ : période de la houle (en s)

Ts : période du jet de rive (en s)

g : accélération de la pesanteur $\left(9,81 \mathrm{~m} \cdot \mathrm{s}^{-2}\right)$

$\beta$ : pente de la plage (en degrés.)

$\mathrm{L}$ : longueur d'onde de la houle à la côte (en $\mathrm{m}$ )

Lo : Longueur d'onde de la houle au large (en m)

Sur le littoral des Bas-Champs, la caractérisation morphodynamique des plages est un travail d'autant plus pertinent que deux morphologies de plages différentes cohabitent (un haut de plage raide à galets, et un bas de plage et une avant-plage à pente faible et à caractère sableux), induisant au cours du cycle tidal des changements brusques et très marqués dans les caractéristiques dynamiques de ces plages. Cette caractérisation est basée sur des observations de terrain couplées à des mesures de divers paramètres morphologiques et dynamiques, notamment la houle. Ces observations ont été réalisées de façon instantanée à intervalles rapprochés mais non réguliers, avec un tachéomètre infrarouge, une échelle de mesure analogique, un chronomètre et un courantomètre-houlographe électromagnétique à capteur de pression de type «S4» (Dolique, 1997, 1998 ; Dolique et Anthony, 1998).

\section{Résultats}

Les résultats exprimés par les six paramètres utilisés sont synthétisés dans la figure 3 . Ces paramètres mettent bien en évidence la nette dichotomie morphodynamique du système plage étudié (DoliQue, 1998). L'ensemble de ces paramètres permet une approche semi-quantifiée de la morphodynamique du système de plage de galets et de bas de plage sableux des Bas-Champs, notamment par la transition marquée des caractéristiques des vagues dans les conditions de déferlement. Transversalement, il y a un passage radical d'un domaine dissipant vers un domaine réfléchissant sur ce système à double entité granulométrique et morphologique. Ce passage s'accompagne de changements importants des caractéristiques des vagues au déferlement, c'est-à-dire que l'on va observer le passage d'une houle déversante à une houle réfléchissante, aspect important dans la dynamique sédimentaire du fait de la différence d'énergie et de capacité de transport entre ces deux types de vagues. Avec les différences de pente, la marée, en déterminant la profondeur d'eau, conditionne les changements temporels du régime morphodynamique, engendrant des variations du niveau de dissipation ou de réflexion et de ce fait, les conditions du déferlement et de jet de rive qui façonnent l'ensemble du système plage des BasChamps.

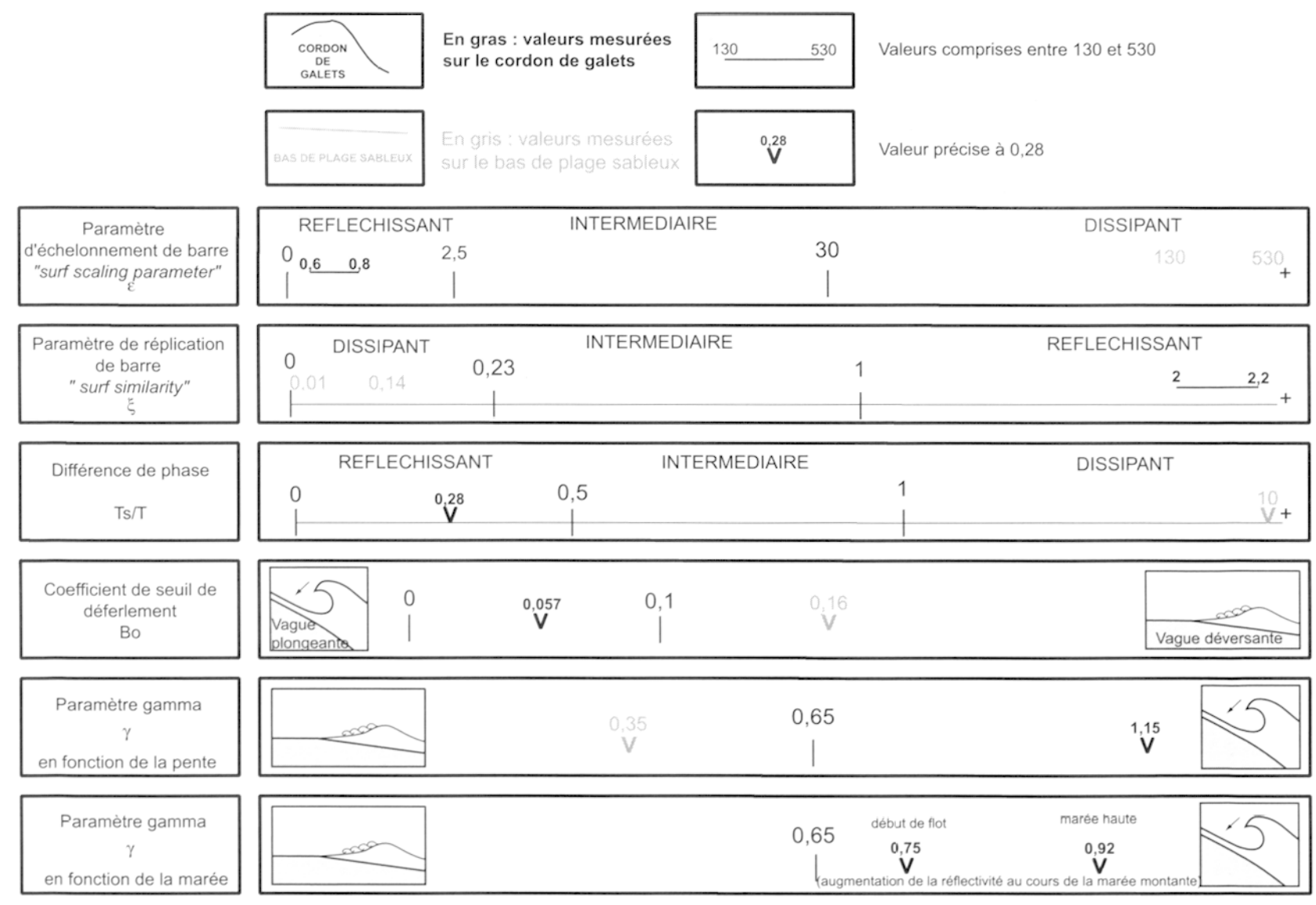

FIG. 3 - RÉSULTATS DE L'UTILISATION DES 6 PARAMÈTRES MORPHODYNAMIQUES CHOISIS 


\section{Conclusion : intérêt de la démarche}

L'approche morphodynamique de l'étude des plages est basée sur l'analyse des ajustements mutuels entre la topographie et les processus hydrodynamiques. Il s'agit d'une approche fine à court terme basée sur une caractérisation semiquantitative et empirique du comportement des plages. Partant de diverses mesures sur les paramètres des houles et de la topographie, cette approche met en évidence en particulier les contrastes importants, mais aussi les articulations dynamiques, entre le bas de plage sableux et plat et le haut de plage de galets raide de la flèche de Cayeux.
Ces outils permettent également de sortir du cadre strictement descriptif afin d'aller vers une différenciation établie à partir de données internationalement reconnues, pour une caractérisation plus objective. L'intérêt est donc finalement très démonstratif et pédagogique. Toutefois, il faut toujours garder à l'esprit les limitations et le côté parfois réducteur de ces paramètres et indices, tant les variables prises en compte, ainsi que leurs interactions, sont complexes et les mécanismes de transfert d'énergie encore mal connus (BAUER et GrEenwOOd, 1988 ; ANTHONY, 1991, 1998).

\section{BIBLIOGRAPHIE}

Anthony E.J., (1991).- Une évaluation des paramètres morphodynamiques couramment utilisés dans la caractérisation des plages, Revue d'Analyse Spatiale Quantitative et Appliquée, 30, p.23-30.

Anthony E.J., (1998).- Sediment-wave parametric characterization of beaches, Journal of Coastal Reaserch, 14-1, p.347-352.

Bauer B.O., Greenwood B., (1988).- Surf-zone similarity, Geog. Rev., 78, p.137-147

CARTER R.W.G. (1988).- Coastal environments, Academic Press, $617 \mathrm{p}$.

Dolique F. (1997).- Caractérisation morphodynamique et sédimentation d'un cordon de galets sous influence estuarienne, Actes du colloque international BORDOMER 97, tome 1, p.231-242.

Dolique F., (1998).- Dynamique morphosédimentaire et aménagements induits du littoral picard au sud de la baie de Somme, Thèse de doctorat en géographie physique, Université du littoral, Dunkerque, $420 \mathrm{p}$.

Dolique F., Anthony E.J., (1998).- The gravel barrier of
Cayeux-sur-Mer, Picardy, France : a summary of recent morphosedimentary changes, Journal de Recherche Océanographique, Vol. 23, $n^{\circ} 4$, p.157-162.

KEMP P.H., (1961).- The relationship between wave action and beach profile characteristics, Proc. $7^{\text {th }}$ Conf. Coast. Eng., Amer. Soc. Civ. Eng., p.262-277.

Kemp P.H., Plinston D.T., (1968).- Beaches produced by waves of low phase difference, Proc. ASCE Journ. Hydr. Div., 94, p.1183-1195.

Short A.D., Hesp P.A., (1982). - Wave, beach and dune interactions in southern Australia, Marine Geology, 48, p.259-284.

Wright L.D., Short A.D., (1983).- Morphodynamics of beach and surf zones in Australia, in KOMAR P.D., Handbook of coastal processes and erosion, CRC Press, Boca Raton, p.35-64.

Wright L.D., Short A.D., (1984).- Morphodynamic variability of surf zones and beaches : a synthesis, Marine geology, 56, p.93-118. 